\title{
Challenges ahead for Polio free India
}

\author{
Sabde Y \\ Dr Yogesh sabde, Professor of Community Medicine, R D Gardi Medical College, Ujjain, MP, India
}

Address for correspondence: Dr Yogesh Sabde, Email: sabdeyogesh@gmail.com

\begin{abstract} still facing in this Polio free era.

Global Polio Eradication Initiative (GPEI) was launched in 1988 based on the resolution of World Health Assembly to target polio for global eradication by 2000 . The strategic components of the resolution were to reach and maintain high levels of routine coverage with oral poliovirus vaccine (OPV), to top up immunization with supplementary doses of OPV, to establish systematic surveillance of polio, and to use targeted mop up campaigns to interrupt any remaining chains of wild poliovirus (WPV) transmission.
\end{abstract}

India has been officially removed from the list of Polio endemic countries a year ahead. It has been a long journey of many years from being Polio endemic to Polio free country. Still work has not been completed. There are many challenges we are

These strategies are translated in to Polio Eradication and Endgame Strategic Plan 2013-2018 to lead a polio free world by $2018^{1}$. OPV was developed in 1961 by Albert Sabin as live, attenuated strains of poliovirus (PV) against all the three PV types. This was called as "trivalent OPV (tOPV)" or "Sabin vaccine" which was safe, effective and economic vaccine to induce long lasting immunity against all the three types of $\mathrm{PV}^{2}$. The efficacy of tOPV was higher for the type 2 component than the other two (type 1 and 2) components. As a result type 2 was not detected in the community acquired infections after 1999. The type 2 component was dropped from the OPV in many countries to introduce bivalent OPV (bOPV) to avoid the possibility of back mutation of the type 2 vaccine strains ${ }^{3}$.

Today polio has been eliminated from most of the countries including India which was officially removed from the list of Polio-endemic countries on February 25, 2012. ${ }^{4}$ This was an important mile stone towards the target of global polio eradication as India is the home for highest number as well as density of population groups which are vulnerable to polio. The achievement of this state in India was delayed by 11 years owing to many biological as well as non biological challenges discussed in the literature. ${ }^{5}{ }^{6}$ During early days of GPEI, it was expected that immunization against poliomyelitis could be stopped after WPV eradication based on the experiences of smallpox eradication in 1977. But the subsequent evidences suggested that the absence of WPV in human circulation alone is not sufficient to declare a "polio free" status. ${ }^{7,8,9}$ The present review will discuss the challenges that India as well as the other countries looking forward for polio eradication after their WPV elimination.

WPV importation: Wild poliovirus (WPV) transmission is still endemic in Afghanistan, Nigeria and Pakistan. All countries including India are at risk of WPV importation from these countries. ${ }^{10}$ Outbreaks of poliomyelitis due to imported WPVs have been reported in 2011 from number of African countries. Examples of countries with WPV importation in last ten years include Chad, Niger, Tajikistan, China and many more. ${ }^{11}$ Such outbreaks were exclusively reported from the countries that used OPV in the polio eradication program. Therefore all such poliofree countries including India are at a risk of re-infection as long as WPV is circulating anywhere in the world. Mop up campaigns, supplementary immunization rounds and heightened surveillance activities of internationally sanctioned standards can successfully stop these importations quickly. ${ }^{12}$

Vaccine-derived poliovirus (VDPVs): The strategies of World Health Assembly (WHA) mainly focused on use of oral poliovirus vaccine (OPV) which contained live (Sabin) strains. ${ }^{1}$ These viruses were known to cause secondary spread and regain their neurovirulence ${ }^{2}$. When vaccine viruses repeatedly replicate in the intestines of unvaccinated children they undergo mutations so that their genetic sequences become similar to those of WPV. Such mutated PVs are called "vaccine-derived polioviruses" (VDPVs). ${ }^{5}$ Three forms of VDPVs are documented viz. circulating (cVDPVs) those related to lower population immunity, immunodeficiency related (iVDPVs) and ambiguous (aVDPVs). ${ }^{13}$ Though the episodes of VDPVs are very rare $^{14}$, VDPVs can remain 
silent for many years and can cause polio outbreaks when transmitted to unvaccinated children ${ }^{5,6}$. VDPVs will be the only source of live polioviruses after elimination of wild polioviruses. Therefore true polio eradication needs absence of transmission of wild as well as vaccine virus.

VDPVs are routinely screened since 2009 by the global polio laboratory network. The recommendation of GPEI so far was to control VDPVs is to immunize every child with OPV several times regardless of the virus (WPV or VDPV). ${ }^{14}$ However cases of VDPVs have been mainly reported in countries using OPV for polio eradication. In 2012 the number of countries reporting VDPVs was more than those reporting WPV outbreaks. On this background current evidences advocate the need to move for phase 2 for polio vaccine virus eradication using inactivated poliovirus vaccine (IPV). IPV vaccination will help to prevent poliomyelitis in IPV vaccinated individuals exposed to VDPVs and WPVs and accelerate wild poliovirus eradication by boosting immunity to WPVs. IPV will also mitigate the risks of VPDVs associated with the withdrawal of OPV type 2. ${ }^{6}$ A few high-income countries had already eliminated polio using only IPV ${ }^{6}$. Therefore polio end game strategic plan highlighted the need for introduction of at least one dose of IPV in all OPV using countries by $2015 .^{15}$

Environmental surveillance of poliovirus: Environmental surveillance for PVs includes monitoring of WPV as well as VDPVs in human circulation through the examination of environmental specimens which are supposed to be contaminated by human faeces. Large proportion of PV infected cases are subclinical that cannot be detected in the routine acute flaccid paralysis (AFP) surveillance.

However PV infected individuals (including subclinical ones) shed large amounts of PV in their faeces for many days after the infection. Environmental surveillance links data from large population groups which are potential reservoirs of PV that are catered by the sewage system thus increasing sensitivity of AFP.

Environmental surveillance is especially important in urban areas and the areas where virus re-introduction is suspected where AFP surveillance is less reliable. ${ }^{9}$ Therefore World Health Organization (WHO) has included environmental surveillance in the new strategic plan for years 2010 -2012. However it has not yet been included as an indicator in the minimum levels for certification standard surveillance. ${ }^{16}$

Care of affected individuals: Acute flaccid paralysis (AFP) surveillance was a part of global polio eradication. AFP surveillance has detected large number of cases suffering from residual post polio paralysis in India. But studies in India report that AFP surveillance has not made sufficient provisions for medical care for the patients. ${ }^{17}$ As per the declaration of Lisbon on rights of patients diagnosis followed by adequate treatment and care are basic right of the patients enshrined. ${ }^{18}$ Ministry of Health of Tajikistan and WHO regional office, built up their rehabilitation capacity to serve APF cases detected after an outbreak of polio in $2010 .{ }^{19}$

Conclusion: If successful, polio would become the second infectious disease to be wiped out after smallpox. However to attain such status the countries like India must get rid of the challenges discussed above. The essential activities that need attention in a polio free world are control of imported WPV, eradication of VDPVs, care of polio affected individuals and environmental surveillance. The major strategic concerns for polio free countries like India will be the planning for withdrawal of OPV type2, introduction of IPV in the polio eradication program.

\section{References}

1. Global Polio Eradication Initiative $>$ About us $>$ Strategy. 2018 (2013), cited 2014 Feb 26.Available from: $<$ http://www.polioeradication.org/Aboutus/Strategy.aspx>

2. Oral polio vaccine ( OPV ). 1-2 (2006), cited 2014 Feb 26. Available from: <http://www.polioeradication. org/ Polioandprevention/Thevaccines/Oralpoliovaccine(OPV). aspx>

3. John, T. J. Two good reasons to drop type 2 virus from. 364, 2004 (2004).

4 Polio Highlight -India Officially Removed from the List of Polio-endemic Countries. 4-6 (2012), cited 2014 Feb 26.Available from: <http://www.paho.org/ hq/index.php? option $=$ com_content $\&$ view $=$ category\&layout $=$ blog\&id $=1$ 875\&Itemid $=2244 \&$ lang $=$ en \&limitstart $=50>$

5. Chumakov, K., Ehrenfeld, E., Wimmer, E. \& Agol, V. I. Vaccination against polio should not be stopped. Nat. Rev. Microbiol. 5, 952-8 (2007).

6. John, T. J. \& Vashishtha, V. M. Eradicating poliomyelitis: India' $\mathrm{s}$ journey from hyperendemic to polio-free status. 137, 881-894 (2013).

7. Adams, P. Ending polio, one type at a time. Bull. World Health Organ. 90, 482-3 (2012).

8. Bhaumik, S. Polio eradication: Current status and challenges. J. Fam. Med. Prim. Care 1, 84 (2012). 
9. Chatterjee, A., Vidyant, S. \& Tn, D. Polio eradication in India: progress , but environmental surveillance and vigilance still needed . PubMed Commons. 14-15 (2013). doi:10.1016/j.vaccine.2012.12.060.

10. Global Polio Eradication Initiative $>$ Infected countries Infected countries. 2010, cited 2014 Feb 26.Available from: <http://www.polioeradication.org/ Infectedcountries.aspx $>$

11. Progress toward interruption of wild poliovirus transmission--worldwide, January 2011-March 2012. MMWR. Morb. Mortal. Wkly. Rep. 61, 353-7 (2012).

12. Global Polio Eradication Initiative $>$ Infected countries $>$ Importation countries Importation countries. 2010 (2010), cited 2014 Feb 26.Available from: $<$ http://www.polioeradication.org/Infectedcountries/Impor tationcountries.aspx

13. Vaccine-derived polioviruses (VDPV). 2009-2010 (2009), cited 2014 Feb 26.Available from: <http://www. polioeradication.org/Polioandprevention/Thevirus/Vaccin ederivedpolioviruses.aspx $>$

14. Circulating vaccine-derived poliovirus 1 (cVDPV) 2000-2013. 1,
15. Objective 2 : Immunization systems strengthening and OPV withdrawal. 51-64 (2013), cited 2014 Feb 26. Available from: <http://www.polioeradication.org/ Portals/0/ Document/Resources/StrategyWork/ PEESP CH6_EN_US.pdf $>$

16. Acute Flaccid Paralysis (AFP) surveillance. 2010 (2010), cited 2014 Feb 26.Available from: <http:// www. polioeradication.org/Dataandmonitoring/ Surveillance. $\operatorname{aspx}>$

17. Yotsu, R. R., Abba, K., Smith, H. \& Das, A. Support for children identified with acute flaccid paralysis under the global polio eradication programme in Uttar Pradesh, India: a qualitative study. BMC Public Health 12, 229 (2012).

18. WMA Declaration of Lisbon on the Rights of the Patient. 1-6 (2005), cited 2014 Feb 26.Available from: $<$ http:/www.wma.net/en/30publications/10policies/14/ind ex.html.pdf?print-media-type\&footer-

right $=[$ page $] /[$ toPage $]>$

19. Polio rehabilitation in Tajikistan. 2014 (2011), cited 2014 Feb 26.Available from: <http://www.euro. who. int/en/ countries/tajikistan/news/news/2011/02/poliorehabilitation-in

\section{How to cite this article?}

Shabde Y. Challenges ahead for Polio free India. Int J Med Res Rev 2014;2(1):74-76, 10.17511/ijmrr.2014.i01.15 